\title{
Scaling of Fluorescence Stokes Shift and Superradiance Coherence Size in Disordered Molecular Aggregates
}

\author{
Vladimir Chernyak, Torsten Meier, ${ }^{\dagger}$ Eugene Tsiper, and Shaul Mukamel* \\ Department of Chemistry, University of Rochester, Rochester, New York 14627, and Department of Physics and \\ Material Sciences Center, Philipps University, Renthof 5, D-35032 Marburg, Germany
}

Received: June 9, 1999; In Final Form: August 23, 1999

\begin{abstract}
Fluorescence line shapes and cooperative spontaneous emission in disordered one-dimensional molecular aggregates are calculated using closed expressions derived by applying the method of optimal fluctuation. A simple scaling relation $L_{\mathrm{s}} \sim \omega_{\mathrm{s}}^{-1 / 2}$ is established between the low temperature disorder-induced Stokes shift $\omega_{\mathrm{s}}$ and the superradiance enhancement factor $L_{s}$. Scaling of the Stokes shift with aggregate size, and the time-dependent Stokes shift are discussed as well. Excellent agreement is obtained with numerical simulations.
\end{abstract}

\section{Introduction}

The dynamics of electronic excitations in molecular crystals, nanostructures (e.g., monolayers and superlattices), $J$-aggregates of cyanine dyes, and biological harvesting antennae complexes ${ }^{1-17}$ can be interpreted in terms of disorder-induced exciton scattering and localization, as well as exciton self-trapping (dynamic localization), which originates from strong vibronic coupling. ${ }^{14,18-30}$ Various spectroscopic measurements, such as absorption, ${ }^{4,5}$ fluorescence, ${ }^{11,12}$ pump-probe, ${ }^{12}$ and photon echo, ${ }^{6,7}$ have been employed for probing exciton motions in molecular aggregates.

In this article we calculate the fluorescence line shape and cooperative spontaneous emission (superradiance) in molecular aggregates using the Frenkel exciton model with strong diagonal disorder. Current theories treat strong static disorder using numerical simulations. ${ }^{10,19,21,22,29,30}$ A similar problem which appears in calculating the density of states in the band tails in disordered semiconductors ${ }^{31}$ has been successfully treated by an elegant method of optimal fluctuation propose independently by Halperin and Lifshitz. ${ }^{32,33}$ We apply this method for calculating fluorescence spectra and obtain a universal scaling relation between the fluorescence Stokes shift and the superradiance factor.

In Section II we derive an analytical asymptotically exact expression for the distribution $n_{L}(\omega)$ of the lowest exciton energy $\omega$ in a disordered aggregate where $\omega$ lies below the band edge of an ordered reference system. Since at low temperatures the fluorescence originates from the lowest exciton state, we can relate the Stokes shift $\omega_{\mathrm{s}}$, defined as the shift between the maxima of the fluorescence and absorption line shapes, to the disorder strength $\sigma$ and the aggregate size $L$ (we assume that the aggregate has a finite size which is large enough to neglect boundary effects). In Section III we apply the optimal fluctuation theory and establish a relation between the Stokes shift $\omega_{\mathrm{s}}$ and superradiance factor $L_{\mathrm{s}}$, which only depends on aggregate geometry (i.e., dimensionality and the magnitude of the intermolecular coupling), and is independent of the magnitude of the disorder. An analytical expression is derived which relates $L_{\mathrm{s}}$ to $\omega_{\mathrm{s}}$ in $1 \mathrm{D}$ aggregates. The scaling of the Stokes

\footnotetext{
* Corresponding author. E-mail: mukamel@chem.rochester.edu.
}

† Philipps University. shift with aggregate size is analyzed in Section IV. We find that for large aggregates the Stokes shift is determined by energy transfer between localized exciton states and may not be obtained from a static model of eigenstates. Our results are summarized in Section V where the time-dependent Stokes shift in disordered aggregates is discussed as well.

\section{Optimal Fluctuation Theory for the Stokes Shift and Superradiance}

We consider an ensemble of one-dimensional molecular aggregates each made out of $L$ coupled two-level molecules with periodic geometry. The Frenkel exciton wave functions $\phi_{m}^{\alpha}$ and energies $\omega_{\alpha}$ are determined by the Schrodinger equation

$$
\sum_{n} J_{m n} \phi_{n}^{\alpha}+\left(\Omega+\xi_{m}\right) \phi_{m}^{\alpha}=\omega_{\alpha} \phi_{m}^{\alpha}
$$

where $n$ and $m$ label individual molecules and the intermolecular coupling $J_{m n}$ describes exciton hopping. The electronic transition frequency of the $m$ th molecules is given by $\Omega+\xi_{m}$ where $\xi_{m}$ represents static diagonal disorder. We assume uncorrelated Gaussian disorder, where the distribution of $\xi_{m}$ is

$$
W(\xi)=\frac{1}{(\sqrt{2 \pi \sigma})^{L}} \exp \left\{-\frac{1}{2 \sigma^{2}} \sum_{m} \xi_{m}^{2}\right\}
$$

In the absence of disorder, $\xi_{m}=0$ and eq 2.1 gives an excitonic band whose width is determined by $J_{m n}$. Disorder changes the exciton energies and leads to the formation of states below the band edge. Assuming that thermal equilibration of excitons is much faster than their radiative lifetime, the main contribution to the fluorescence at low temperatures comes from the lowest exciton. The position of the lowest exciton thus determines the electronic Stokes shift of the fluorescence, whereas the superradiant factor is related to the shape of the corresponding exciton wave function. Both the lowest energy level and its wave function have a statistical nature and the fluorescence spectrum is obtained by averaging over all possible realizations of disorder

$$
f(\omega)=L_{\mathrm{s}}(\omega) n_{L}(\omega)
$$


Here $n_{L}(\omega)$ is the lowest state energy distribution, and the superradiant enhancement factor $L_{\mathrm{S}}(\omega)$ is the radiative decay rate relative to a single molecule. In general eq 2.3 should contain the average of product rather than the products of averages of both quantities. The factorization employed here is justified, as will be verified by our numerical simulations.

To find $n_{L}(\omega)$ we introduce the probability $F(\omega)$ that there is a level below $\omega$. Since low-energy excitons are localized and do not communicate, this probability thus satisfies Poisson statistics

$$
F(\omega)=1-\exp \left(-L \int_{-\infty}^{\omega} \mathrm{d} \epsilon n(\epsilon)\right)
$$

where $L$ is the number of molecules in the aggregate, and $n(\epsilon)$ is the normalized exciton density of states (i.e., $\int_{-\infty}^{\infty} \mathrm{d}_{\omega} n(\omega)=1$ ). Since the statements "the lowest exciton level lies below $\omega$ " and "there is an exciton level below $\omega$ " are equivalent, $F(\omega)$ also describes the probability for the lowest level to lie below $\omega$. This implies $n_{L}(\omega)=\mathrm{d} F(\omega) / \mathrm{d} \omega$ which by making use of eq 2.4 yields the following expression for $n_{L}(\omega)$ in terms of the density of states

$$
n_{L}(\omega)=L n(\omega) \exp \left[-L \int_{-\infty}^{\omega} \mathrm{d} \epsilon n(\epsilon)\right]
$$

A more careful derivation shows that eqs 2.5 and 2.3 hold provided the high-order level correlation functions (multidimensional density of states) can be factorized for energies below $\omega$, which is asymptotically true when $\omega$ is sufficiently low. This can be done using the exact relation

$$
n_{L}(\omega)=L\left\langle\hat{n}(\omega) \exp \left(-L \int_{-\infty}^{\omega} \mathrm{d} \epsilon \hat{n}(\epsilon)\right)\right\rangle
$$

where the average in the rhs of eq 2.6 should be understood as

$$
\left\langle\hat{n}\left(\epsilon_{1}\right) \ldots \hat{n}\left(\epsilon_{j}\right)\right\rangle=n^{(j)}\left(\epsilon_{1}, \ldots, \epsilon_{\mathrm{j}}\right)
$$

and $n^{(j)}$ are the multi-level correlation functions.

A closed expression for the lowest state energy distribution $n_{L}(\omega)$ can be obtained by substituting into eq 2.5 the exact expression for $n(\omega)=d N(\omega) / \mathrm{d} \omega$ where the cumulative density of states is given by

$$
N(\omega) \equiv \int_{-\infty}^{\omega} \mathrm{d} \epsilon n(\epsilon)
$$

Hereafter we choose the zero energy to correspond to the bottom of the exciton band in an ordered reference system. For $1 \mathrm{D}$ systems with nearest-neighbor hopping, i.e., $J_{n, n+1}=J_{n+1, n}$ $=-J$, in the continuum limit we have $\mathrm{e}^{34,35}$

$$
N(\omega)=\frac{2}{\pi}\left(\frac{\sigma_{0}}{J}\right)^{1 / 2}\left[A_{i}^{2}\left(-\omega / \sigma_{0}\right)+B_{i}^{2}\left(-\omega / \sigma_{0}\right)\right]^{-1}
$$

Here $A_{i}$ and $B_{i}$ are the Airy functions ${ }^{36}$ and $\sigma_{0}=\sigma(\sigma / 4 J)^{1 / 3}$. The asymptotic $\omega \rightarrow-\infty$ expression for $N(\omega)$ which follows from eq 2.9 and has the form

$$
N(\omega)=\frac{2}{\pi}\left(\frac{|\omega|}{J}\right)^{1 / 2} \exp \left[-\frac{4}{3}\left(\frac{|\omega|}{\sigma_{0}}\right)^{3 / 2}\right]
$$

Equation 2.10, together with eqs 2.5 and 2.3, describe the low-temperature relaxed fluorescence signal. Equation 2.10 can be alternatively obtained using the optimal fluctuation method as discussed below.
The superradiance factor $L_{\mathrm{s}}$, defined as the ratio of the radiative decay rate of an aggregate to that of a single chromophore, is given by ${ }^{29}$

$$
L_{\mathrm{s}}(\omega)=\sum_{m n} M_{m n} \rho_{m n}(\omega)
$$

where $M_{m n} \equiv \mathbf{d}_{\mathrm{m}} \cdot \mathbf{d}_{\mathrm{n}}$ is the window function expressed in terms of the unit vectors $\mathbf{d}_{\mathrm{m}}$ in the direction of the single chromophore transition dipole moments and $\rho_{m n}$ is the thermally relaxed single-exciton density matrix at zero temperature, given by

$$
\rho_{m n}=\sum_{j} \phi_{m+j}^{(0)} \phi_{n+j}^{(0)}
$$

where the summation over $j$ reflects the fact that $\phi^{(0)}$ can be centered at any chromophore.

The method of optimal fluctuation ${ }^{31-33}$ is based on the observation that the conditional probability $W(\xi ; \omega)$ of a disorder configuration $\xi$ subject to the constraint that the lowest exciton has energy $\omega$, is sharply peaked at $\xi=\xi^{(0)}$, known as the optimal fluctuation, provided the exciton energy $\omega$ is far below the exciton band edge. For large aggregates the lowest energy state lies far below the band edge for most of the aggregates in the ensemble. This implies that the wave function $\phi^{(0)}$ of the emitting state in a large aggregate is given by the solution of eq 2.1 for $\xi_{n}=\xi_{n}{ }^{(0)}$. To determine $\phi^{(0)}$, we find the extremum of $W(\xi)$ for a fixed exciton energy (which is equivalent to finding the extremum of $W(\xi)-g \epsilon(\xi)$, where $\epsilon(\xi)$ is the lowest exciton energy and $g$ is the Lagrange multiplier). Making use of $\partial \epsilon / \partial \xi_{j}=-\left|\phi_{j}\right|^{2}$ (which is a direct consequence of perturbation theory, ${ }^{37}$ we obtain $\xi_{j}=-g\left|\phi_{j}\right|^{2}$, which yields upon the substitution into eq 2.1

$$
\begin{gathered}
\sum_{n} h_{m n} \phi_{n}^{(0)}-\omega \phi_{m}^{(0)}-g\left|\phi_{m}^{(0)}\right|^{2} \phi_{m}^{(0)}=0 \\
\sum_{m}\left|\phi_{m}^{(0)}\right|^{2}=1
\end{gathered}
$$

with $h_{m n}=\left(\Omega-\Omega_{0}\right) \delta_{m n}+J_{m n}$, where $\Omega_{0}$ is the exciton band edge (we reiterate that the zero energy corresponds to the exciton band edge in an ordered reference system). The wave function $\phi_{n}{ }^{(0)}$ is obtained by solving eq 2.13 whereas the Lagrange multiplier $g$ is determined by the normalization condition eq 2.14. We note that the same system of equations 2.13 and 2.14 determines the superradiance factor $L_{\mathrm{S}}$ for polaron control of excitonic coherence in the case of diagonal vibronic coupling in the adiabatic limit. ${ }^{29}$ Static disorder and vibronic coupling thus lead to the same relation between the superradiance factor and the Stokes shift. However, there is one important difference: in the polaron case in eq $2.13 \mathrm{~g}$ represents vibronic coupling and the Stokes shift is obtained by solving the system of equations. In the present case of static disorder $g$ represents a Lagrange multiplier whereas $\omega$ is the lowest exciton energy and should be given as an input.

Since, as will be demonstrated below, for large aggregates $\rho_{m n}(\omega)$ changes slowly in the region where $n_{L}(\omega)$ has a sharp peak, the Stokes shift is determined by the position of the maximum of the lowest state energy distribution $n_{L}(\omega)$. The maximum of the optical absorption corresponds to the bottom of the exciton band in the reference ordered system $\omega=0$ (according to our convention). This implies that at low temperatures the Stokes shift is given by the lowest exciton energy $\omega_{\mathrm{s}}=\omega$. Eqs 2.13 and 2.14, together with eqs 2.11 and 2.12, establish the relation between the Stokes shift and the super- 
radiance factor: solving eq 2.13 for $\omega=\omega_{\mathrm{s}}$, yields $\phi^{(0)}$, which determines $L_{\mathrm{s}}$ upon substitution into eq 2,12. In the next section we use these results to derive a closed analytical relation between the Stokes shift and the superradiant factor of 1D aggregates.

\section{Comparison with Numerical Simulations}

We consider 1D aggregates with fixed parallel orientation of the molecular transition dipoles (e.g., J-aggregates). The generalization to circular aggregates such as light-harvesting systems and 2D monolayers is straightforward. We present analytical expressions in the continuum limit and compare with simulations. We start by deriving closed expressions for $\omega_{\mathrm{s}}$ and $L_{\mathrm{s}}$. If for the relevant energies the exciton localization length is much smaller than the system size and much larger than the lattice constant, eqs 2.13 and 2.14 can be solved in the continuum limit, which yields the Ginzburg-Landau soliton for the wave function ${ }^{29}$

$$
\phi(n)=\left(2 a_{0}\right)^{-1 / 2} \operatorname{sech}\left(n / a_{0}\right)
$$

with $a_{0}=4 J / g$ and $\omega=-J / a_{0}^{2}$, where $-J$ is the nearestneighbor intermolecular coupling. The parameter $a_{0}$ is defined by the energy of the localized state which gives the Stokes shift. The solution given by eq 3.1 corresponds to the continuum limit of an infinite system. Equations 2.13 and 2.14 can be solved analytically in the continuum limit in a periodic system. The solution in this case is expressed in terms of elliptic functions. ${ }^{38}$ When the localization length is comparable with the lattice constant, eqs 2.13 and 2.14 should be solved numerically.

For parallel dipole orientation, $M_{m n}=1$, and the substitution of $\phi(n)$ into eqs 2.12 and 2.11 and replacing the sum in eq 2.12 by an integral yields

$$
L_{\mathrm{s}}=\frac{\pi^{2}}{2}\left(\frac{J}{\left|\omega_{\mathrm{s}}\right|}\right)^{1 / 2}
$$

Note that this simple scaling relation between the superradiant factor $L_{\mathrm{s}}$ and the Stokes shift $\omega_{\mathrm{s}}$ is independent of the magnitude of disorder.

Using these expressions we have calculated the lowest exciton distribution $n_{L}(\omega)$, the fluorescences spectrum $f(\omega)$, and the superradiant factor $L_{\mathrm{s}}(\omega)$ of the lowest exciton in a cyclic aggregate with the parallel dipole orientation which consists of $L=18$ chromophores, resembling the LH2 harvesting complex of purple bacteria. ${ }^{12}$

Figure 1 shows $n_{L}(\omega)$ (upper panel), $f(\omega)$ (middle panel), and $L_{\mathrm{S}}(\omega)$ (lower panel) for a nearest-neighbor coupling of $J=34.7 \mathrm{meV}$ and $\sigma=23.5 \mathrm{meV}$. The dashed and dotted lines in the upper and middle panels display $n_{L}(\omega)$ obtained using eq 2.5 and $f(\omega)$ (eq 2.3), where $n(\omega)$ is taken from eq 2.10 and 2.9 , respectively. The dashed line in the lower panel displays $L_{\mathrm{s}}(\omega)$ computed using eq 3.2 . To test the validity of the approximate analytical expressions we have computed the same quantities by direct numerical simulations. Simulations are averaged over 1 million disorder realizations. The direct numerical results given by the solid lines in all panels show an excellent agreement with the red part of the fluorescence spectra up to its maximum. The zero of the energy scale coincides with the energetic position of the lowest exciton in the absence of disorder. The dashed dotted lines in the upper and middle panels display $n_{L}(\omega)$ (eq 2.5) and $f(\omega)$ (eq 2.3) taking $n(\omega)$ directly from the simulations. Figures 2 and 3 display the same quantities

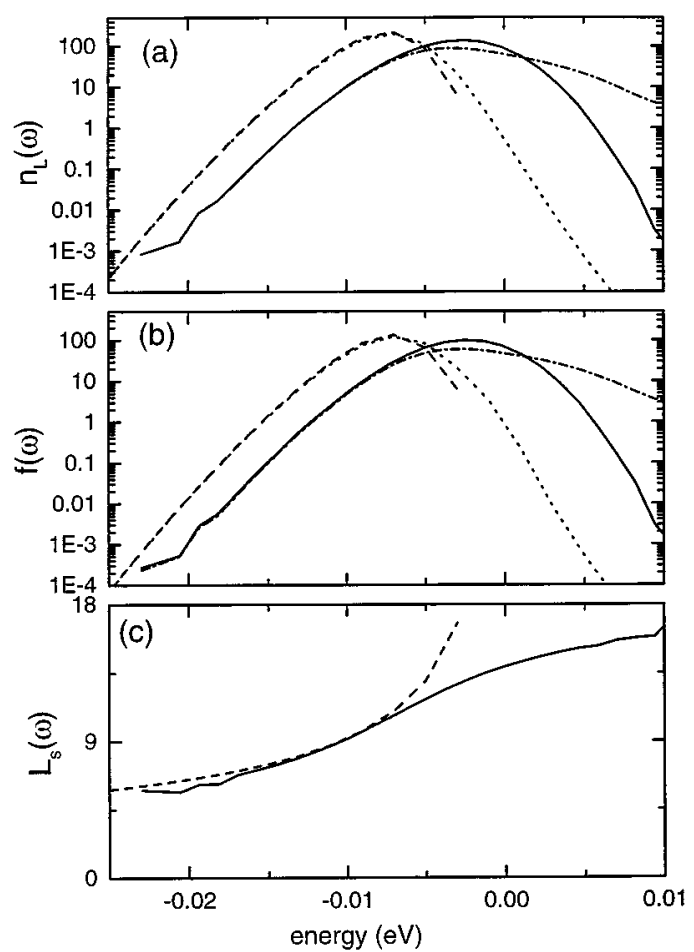

Figure 1. Upper panel-the distribution of lowest-energy exciton $n_{L^{-}}$ $(\omega)$; middle panel-the low-temperature fluorescence spectrum $f(\omega)$; lower panel-the superradiant factor $L_{\mathrm{s}}(\omega)$ for a cyclic disordered aggregate with $L=18$ chromophores with nearest-neighbor coupling of $J=34.7 \mathrm{meV}$ considering Gaussian, uncorrelated, diagonal disorder (see eq 2.2) with $\sigma=23.5 \mathrm{meV}$. The zero of the energy scale coincides with the energetic position of the lowest exciton in the absence of disorder. The solid lines are the results of direct numerical simulations. The dashed and dotted lines in the upper and middle panels display $n_{L}(\omega)$ using eq 2.5 and $f(\omega)$ using eq 2.3, where $n(\omega)$ is taken from eqs 2.10 and 2.9, respectively, and the dashed dotted lines display the same quantities using $n(\omega)$ taken directly from the simulations. The dashed line in the lower panel displays $L_{\mathrm{s}}(\omega)$ according to eq 3.2.

for different values of the disorder parameter $\sigma=70.5$ and $\sigma$ $=117.5 \mathrm{meV}$, respectively. The agreement with simulations is similar to Figure 1.

Our analytical expression does not apply to the blue part of the fluorescence spectra since this spectral region lies in the vicinity of the band edge of the ordered reference system, where the factorization of the higher-order level correlation functions (eq 2.7) is not justified and the optimal fluctuation theory is inapplicable. We note that already for $L=18$ the analytical theory reproduces the red half of the fluorescence spectra. When $L$ is increased further the spectra are red-shifted, which increases the range of applicability of the theory. It is important to note that a good agreement is obtained only if we use eq 2.5 for $n_{L}(\omega)$ with $n(\omega)$ obtained from a simulation rather then from eq 2.9 or eq 2.10. This can be rationalized as follows. Eq 2.5 contains the density of states $n(\epsilon)$ in the exponent with a large pre-factor of $L . n_{L}(\omega)$ is therefore extremely sensitive to small deviations of $n(\epsilon)$ from its exact value. In the case of eqs 2.9 and 2.10 these deviations originate from the continuum approximation. For low energies, however, the analytical expressions predict the energy dependence of $n_{L}(\omega)$ and $f(\omega)$ as obtained from the simulations.

\section{Scaling of the Stokes Shift with Aggregate Size}

To investigate the variation of the Stokes shift with aggregate size we note that at low temperatures the fluorescence originates 


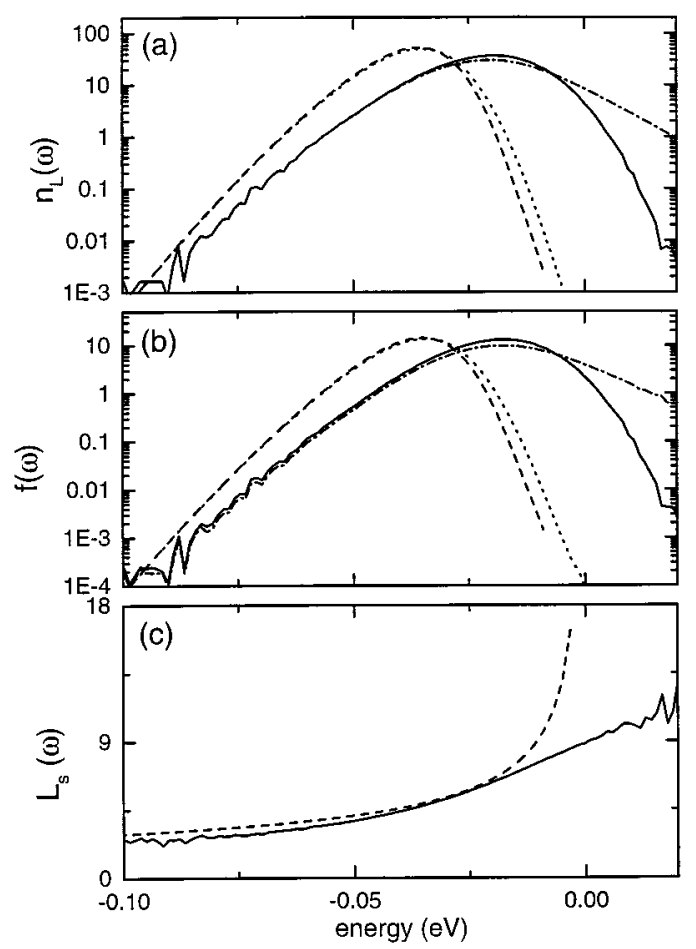

Figure 2. Same as Figure 1 but for $\sigma=70.5 \mathrm{meV}$.
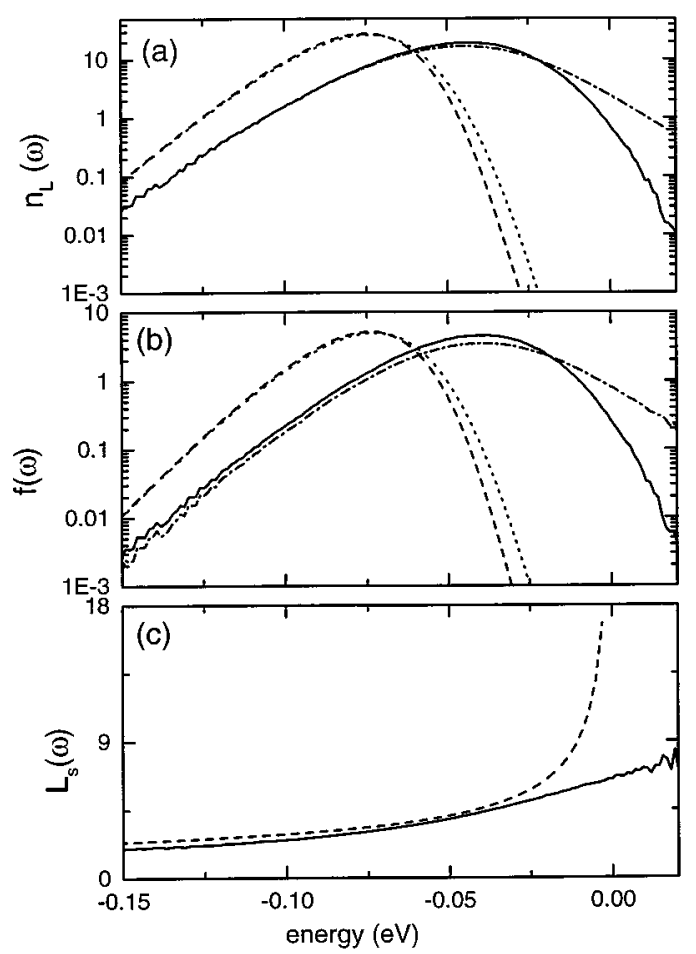

Figure 3. Same as Figure 1 but for $\sigma=117.5 \mathrm{meV}$.

from the lowest exciton. The Stokes shift can therefore be obtained by finding the maximum of the distribution of the lowest energy state $n_{L}(\omega)$, given by eq 2.5 . Alternatively, the Stokes shift can be estimated using the following argument: the average number of states with energies below $\omega$ is $L N(\omega)$, where $N(\omega)$ (eq 2.9) is the cumulative density of states. The Stokes shift $\omega_{\mathrm{s}}$ can then be estimated by requiring $L N\left(\omega_{\mathrm{s}}\right)=1$, i.e., the number of one-exciton states with energies below $\omega_{\mathrm{s}}$ should be on the order of one. The $L$-dependent $\omega_{\mathrm{s}}$ can thus be obtained by solving the equation

$$
\mathrm{N}\left(\omega_{\mathrm{s}}\right)=L^{-1}
$$

Since $N(\omega)$ is a monotonically increasing function, eq 4.1 predicts a monotonic decrease of $\omega_{\mathrm{s}}$ with aggregate size $L$. This is unphysical since in a sufficiently large aggregate an exciton does not have enough time to reach this state during its lifetime $\tau$. The Stokes shift is then controlled by the dynamics of energy transfer between low-energy localized exciton states. This leads naturally to a new coherence size $L_{0}$ defined as the size of the segment on which an exciton has enough time to reach the lowest-energy state. In Appendix A we derive an expression for $L_{0}$

$$
L_{0}=\left(J \tau_{0}\right)^{1 / 3}
$$

where $\tau_{0}$ is the radiative lifetime of a single chromophore. For $L<L_{0}$ the Stokes shift is determined by eq 4.1. For $L>L_{0}$ it becomes independent of size and is obtained by the solution of $N\left(\omega_{\mathrm{s}}\right)=L_{0}^{-1}$. For typical $J$-aggregate with $J \sim 300 \mathrm{~cm}^{-1}$, $\tau_{0} \sim 10 \mathrm{~ns}$, we have $L_{0} \sim 100$.

Note that our theory is based on the factorization of the multidimensional densities of states for energies lower than $\omega_{\mathrm{s}}$. This is justified provided the distance between optimal fluctuations $L_{0}(\omega)$ is much larger than the exciton localization length $l_{A}$. Our previous numerical simulations have shown $l_{A} \sim L_{\mathrm{S}} / 3 .^{29}$ The maximum superradiant factor observed experimentally reaches $L_{\mathrm{S}} \sim 50,{ }^{11}$ which gives $l_{A} \sim 15$. Our theory thus applies to disordered $J$-aggregates. Another important consequence of these estimates is that numerical simulations of equilibrated low-temperature fluorescence spectra in disordered aggregates with size $L>100$ will in general be unrealistic since the lowest-energy exciton states in such aggregates are not accessible. The same applies to pump-probe spectra with a long time delay between pump and probe.

The present analysis did not take energy-transfer between aggregates into account. This can be accounted for by replacing $L$ in eq 4.1 by $L_{1}$ defined as the number of chromophores in the volume of size $L_{0}$. This may increase substantially the Stokes shift for closely packed aggregates.

\section{Discussion}

Equations 2.11-2.14 and 4.1 constitute a closed system of equations which express both the superradiant factor and the Stokes shift in terms of the density of states $n(\omega)$. The asymptotic form of the density of states $n(\omega)$ which enters eq 2.5 can be obtained by applying the method of optimal fluctuation. In contrast to $\rho_{m n}$ which is expressed in terms of the solution of eqs 2.13 and 2.14 alone, $n(\omega)$ also involves calculation of the determinant of the linear operator which originates from the integration over $\mathrm{d} \xi$, in the vicinity of $\xi=\xi^{(0)}$, when the integrand is approximated by a Gaussian. In 1D systems the determinant can be evaluated analytically; otherwise it should be computed numerically. The red wing of the fluorescence spectrum all the way to its maximum is well described by eqs 2.3 and 2.5 even for $L=18$.

To obtain a universal estimate for the coherence size (eq 4.2) we have used a simple model by setting $\Gamma(\omega)=V(\omega)$ (see Appendix A for the definitions of $\Gamma$ and $V$ ). The transfer rate $\Gamma(\omega)$ in a realistic situation depends on the vibronic coupling. When the coupling $V(\omega)$ is sufficiently small (which is always true for sufficiently low energy $\omega$ ) the transfer rate $\Gamma(\omega)$ can be calculated using Forster's theory, which yields $\Gamma(\omega)=$ $\left.\left[V(\omega)^{2}\right] / \Delta\right]$ where $\Delta$ is determined by the spectral density of the exciton-bath coupling. The latter can be readily calculated 
for a given model of the chromophore-bath coupling, since the shape of the exciton wave function is known. This together with eq A3 could give a closed expression for $\Gamma(\omega)$ for a general model of the chromophore-bath coupling. Such analysis goes, however, beyond the scope of this article.

We emphasize that the fact that an excitation does not have enough time to reach the lowest state of a large aggregate strongly affects the Stokes shift, and leads to its saturation for aggregates larger than the coherence size $L_{0}$. The relation between $L_{\mathrm{s}}$ and $\omega_{\mathrm{s}}$, however, is not affected provided there is enough time for an excitation to reach an exciton whose energy is sufficiently low, so that optimal fluctuation theory is applicable.

We have addressed the low-temperature fluorescence in disordered molecular aggregates when the effects of disorder are most pronounced. When the temperature $T$ is increased but the main contribution to the signal comes from several lowest states, one can still use the optimal fluctuations theory to find the profile of $\xi_{n}$. Finding the exciton states for this optimal realization of disorder and weighing the exciton contributions with the Boltzmann distribution one obtains $L_{\mathrm{S}}$ for finite $T$. This approach breaks down when temperature effects in the formation of $L_{\mathrm{s}}$ become stronger than their disorder-induced counterparts. In that case the effects of disorder may be neglected and $L_{\mathrm{S}}$ can be found by performing calculations in an ordered reference system.

Finally we note that the optimal fluctuation approach can also be applied to calculate the time-dependent Stokes shift. Since we know the distribution of optimal fluctuations and the coupling $V$ between them, exciton transport can be simulated in a straightforward way by a Monte Carlo sampling of the distribution of the optimal fluctuations. Making some rough approximations such as neglecting the memory allows us to derive simple equations for the time-evolution of the Stokes shift. For example, assuming that the energy transfer occurs with rate $\Gamma(\omega)$ into all accessible energies lower than $\omega$, the time derivative of the Stokes shift can be estimated as $[\Gamma(\omega)]^{-1} \epsilon(\omega)$, where

$$
\epsilon(\omega)=[N(\omega)]^{-1} \int_{-\infty}^{\omega} \mathrm{d} \omega_{1} N\left(\omega_{1}\right)
$$

is the average value of the energy with for the states lying below $\omega$. This yields for the time-dependent Stokes shift

$$
\mathrm{d} \omega_{\mathrm{s}} / \mathrm{d} \tau=-\epsilon\left(\omega_{\mathrm{s}}\right)\left[\Gamma\left(\omega_{\mathrm{s}}\right)\right]^{-1}
$$

where $\Gamma(\omega)$ can be calculated using $V(\omega)$ (eq A3) for an arbitrary model of chromophore-bath coupling.

Acknowledgment. This paper is dedicated to Kent Wilson whose creativity and infectious enthusiasm have always been a constant source of inspiration. The support of the National Science Foundation and the United States Air Force office of scientific research is gratefully acknowledged.

\section{Appendix A. Exciton Dynamics Control of Fluorescence}

In this appendix we consider the coherence size $L_{0}$, and evaluate it for 1D $J$-aggregates. Since all exciton states in a disordered 1D aggregate are localized, only hopping transport induced by vibronic coupling takes place. If an exciton occupies a localized state with energy $\omega$, at low temperature it can typically hop to a state with lower energy thus leading to a timedependent Stokes shift. To find the long-time Stokes shift we assume that the relaxed fluorescence originates from the state for which the transfer time to a lower energy becomes comparable to the exciton lifetime $\tau: \Gamma(\omega) \tau=1$ where $\Gamma(\omega)$ is the energy relaxation rate of an exciton with energy $\omega$.

To find $\Gamma(\omega)$ we first determine the coupling $V(\omega)$ between the states participating in the energy transfer. For making a simple estimate we set $\Gamma(\omega)=V(\omega)$. To determine $V(\omega)$ we note that the number of exciton states with energy smaller than $\omega$ is $L N(\omega)$, which implies that a typical distance between the states participating in the transfer process is $L_{0}(\omega)=[N(\omega)]^{-1}$. This yields for the coherence size $L_{0}=L_{0}(\omega)$. Since both states are formed by optimal fluctuations, their wave functions are known (eqs 2.13 and 2.14) and the coupling can be found in a standard way. ${ }^{39}$ We should, however, be careful when implementing the nearest-neighbor approximation for intermolecular coupling. For $J$-aggregates it has a dipole-dipole form with the dipoles oriented along the chain which yields $J_{m n}=J / \mid m-$ $\left.n\right|^{3}$. This implies that nearest-neighbor hopping constitutes a good approximation for determining the exciton wave function in the region of the trap, but it fails to describe the long-distance asymptote of the wave function which determines the coupling $V(\omega)$. In particular, in the nearest-neighbor case the asymptotic behavior shows exponential decay of the wave function whereas in the dipole-dipole case it is long range. The coupling $V$ between two states localized in different traps (1) and (2) in the long-range hopping case is given by a simple expression

$$
V=\sum_{m n} \phi^{(1)}(m) J_{m n} \phi^{(2)}(n)
$$

where the wave functions $\phi^{(1)}(m)$ can be determined by solving the Schrödinger equation with nearest-neighbor hopping. Eq A1 gives the coupling to first-order in the long-range hopping.

Equation A1 can be also derived formally by evaluating the long-distance asymptote of the wave function and making use of the well-known expression for $V$ in terms of the overlap of wave functions. ${ }^{39}$ When the exciton size is smaller than the distance between the traps, which, as outlined above, is given by $L_{0}$, we can substitute $J / L_{0}^{3}$ for $J_{m n}$ in eq A1 to obtain

$$
V=J L_{0}^{-3}\left[\sum_{m} \phi^{(1)}(m)\right]\left[\sum_{n} \phi^{(2)}(n)\right]
$$

Substituting for $\phi^{(1)}$ and $\phi^{(2)}$ the solution of eqs 2.13 and 2.14 (eq 3.1) and recalling the definition of the superradiant factor (2.11 and 2.12) we obtain from eq A2

$$
V(\omega)=J L_{\mathrm{s}}(\omega)\left[\mathrm{L}_{0}(\omega)\right]^{-3}
$$

Since $L_{0}(\omega)=[N(\omega)]^{-1}$, eq A3 constitutes a closed expression for the coupling between the states at the energy $\omega$.

As outlined before, the coherence size $L_{0}$ is given by $L_{0}(\omega)$, and eq A 3 allows us to find $L_{0}$ by setting $\omega=\omega_{\mathrm{s}}$. Since in our model $\Gamma\left(\omega_{\mathrm{s}}\right)=V\left(\omega_{\mathrm{s}}\right)$ and $\Gamma\left(\omega_{\mathrm{s}}\right) \tau=1$ where the radiative lifetime $\tau$ is related to that of a single chromophore $\tau_{0}$ through the superradiant factor $L_{\mathrm{s}}: \tau=\tau_{0} L_{\mathrm{s}}^{-1}$, eq A3 yields at $\omega=\omega_{\mathrm{s}}$, $L_{\mathrm{s}} \tau_{0}^{-1}=J L_{\mathrm{s}} L_{0}^{-3}$, which gives eq 4.2. The superradiant factor $L_{\mathrm{s}}$ is canceled out and eq 4.2 expresses the coherence size $L_{0}$ in terms of the intermolecular coupling and the radiative lifetime of a single chromophore. The Stokes shift in large aggregates $\left(L \gg L_{0}\right)$ can be found by combining eq 4.2 with the relation $N\left(\omega_{\mathrm{s}}\right)=L_{0}^{-1}$.

\section{References and Notes}

(1) van Grondelle, R.; Dekker, J. P.; Gillbro, T.; Sundstrom, V. Biochim. Biophys. Acta 1994, 1187, 1. 
(2) Sundstrom, V.; van Grondelle, R. In Anoxygenic Photosynthetic Bacteria; Blankenship, R. E., Madiag, M. T., Baner, C. E., Eds.; Kluwer Academic: Dordrecht, The Netherlands, 1995); p 349.

(3) McDermott, G.; Prince, S. M.; Freer, A. A.; HawthornthwaiteLawless, A. M.; Papiz, M. Z.; Cogdell, R. J.; Issacs, N. W. Nature 1995, $374,517$.

(4) Sauer, K.; Cogdell, R. J.; Prince, S. M.; Freer, A. A.; Issaca, N. W.; Scheer, H. Photochem. Photobiol. 1996, 64, 564.

(5) Alden, R. G.; Johnson, E.; Nagarajan, V.; Parson, W. W.; Law, C. J.; Cogdell, R. J. J. Phys. Chem. B 1997, 101, 4667.

(6) Joo, T.; Jia, Y.; Yu, J.-Y.; Jonas, D. M.; Fleming, G. R. J. Phys. Chem. 1996, 100, 2399.

(7) Jimenez, R.; van Mourik, F.; Fleming, G. R. J. Phys. Chem. B 1997, 101,7350

(8) Reddy, N. R. S.; Small, G. J.; Seibert, M.; Picorel, R. Chem. Phys. Lett. 1991, 181, 391. 9458 .

(9) Reddy, N. R. S.; Picorel, R.; Small, G. J. J. Phys. Chem. 1992, 96,

(10) Fidder, H.; Knoester, J.; Wiersma, D. A. Chem. Phys. Lett. 1990, 171, 529. Durrant, J. R.; Knoester, J.; Wiersma, D. A. Chem. Phys. Lett. 1992, 222, 450 .

(11) Özçelik, S.; Özçelik, I.; Akins, D. Appl. Phys. Lett. 1998, 73, 1949; Özçelik, S.; Akins, D. Appl. Phys. Lett. 1997, 71, 3057; Özçelik, S.; Akins, D. J. Phys. Chem. 1997, 101, 3021.

(12) Muneter, A. A.; Brumbaugh, D. V.; Apolito, J.; Horn, L. A.; Spano, F. C.; Mukamel, S. J. Phys. Chem. 1992, 96, 2748. Lanzfame, J. M.; Muneter, A. A.; Brumbaugh, D. V. Chem. Phys. 1996, 210, 79.

(13) de Boer, S.; Wiersma, D. A. Chem. Phys. Lett. 1990, 165, 45. van Burgel, M.; Wiersma, D. A.; Duppen, K. J. J. Chem. Phys. 1995, 102, 20.

(14) Zaitsev, A. I.; Malyshev, V. A.; Trifonov, E. D. Sov. Phys. JETP 1983, 57,275

(15) Kopelman, R. In Spectroscopy and Excitation Dynamics of Condensed Molecular Systems; North-Holland: Amsterdam, The Netherlands, 1983.

(16) Johnson, C. K.; Small, G. J. Excited States; Academic Press: New York, 1982; Vol. 6

(17) Burland, M. S.; Zewail, A. H. Adv. Chem. Phys. 1979, 40, 369.
(18) Grad, J.; Hernadez, G.; Mukamel, S. Phys. Rev. A. 1988, 37, 3838

(19) Spano, F. C.; Mukamel, S. J. Chem. Phys. 1989, 91, 683.

(20) Sundstrom, V.; Gillbro, T.; Gadonas, R. A.; Piskarskas, A. J. Phys. Chem. 1988, 89, 2754.

(21) Chachisvilis, M. Ph.D. Thesis, Lund University, 1996. Chachisvilis, M.; Westerhuis, W.; Hunter, C. N.; Sundstrom, V. J. Phys. Chem. B 1997 101,7275

(22) Monshouwer, R.; Abrahamson, M.; van Mourik, F.; van Grondelle, R. J. Phys. Chem. B 1997, 101, 7241 .

(23) Wang, N.; Muenter, A. A.; Mukamel, S. J. Phys. Chem. 1993, 99 3604; Wang, N.; Chernyak, V.; Mukamel, S. Phys. Rev. B 1994, 50, 5609.

(24) Leegwater, J. A.; Mukamel, S. Phys. Rev. A 1992, 46, 452.

(25) Knoester, J.; Mukamel, S. Phys. Rep. 1991, 205, 1.

(26) Malyshev, V. A. Opt. Spectrosc. 1991, 71, 505; J. Lumin 1993, $55,225$.

(27) Leegwater, J. A. J. Phys. Chem. 1996, 100, 14403.

(28) Chernyak, V.; Wang, N.; Mukamel, S. Phys. Rep. 1995, 263, 213.

(29) Meier, T.; Zhao, Y.; Chernyak, V.; Mukamel, S. J. Chem. Phys. 1997, 107, 3876

(30) Meier, T.; Chernyak, V.; Mukamel, S. J. Phys. Chem. B 1997, 101, 7332.

(31) Shklovskii, B. I.; Efros, A. L. Electronic Properties of Doped Semiconductors; Springer-Verlag: Berlin, 1984.

(32) Halperin, B. I.; Lax, M. Phys. Rev. 1966, 148, 722; Phys. Rev. 1967, 153,802

(33) Lifshitz, I. M. Zh. Eksp. Teor. Fiz. 1967, 53, 743; Sov. Phys. JETP

1968, 26, 462

(34) Efetov, K. B. Adv. Phys. A 1983, 32, 53.

(35) Halperin, B. I. Phys. Rev. A 1965, 139, 104.

(36) Abramowitz, M.; Stegun, I. A. Handbook of Mathematical Functions; Dover: New York, 1972.

(37) Landau, L. D.; Lifshitz, I. M. Quantum Mechanics; Pergamon: New York, 1960

(38) Rashba, E. I. Synth. Met. 1994, 64, 255.

(39) Anselm, A. Introduction to Semiconductor Theory; Prentice Hall: New York, 1982 